Document downloaded from:

http://hdl.handle.net/10251/63422

This paper must be cited as:

Tobón, Jl.; Paya Bernabeu, JJ.; Borrachero Rosado, MV.; Restrepo Baena, OJ. (2012). Mineralogical evolution of Portland cement blended with silica nanoparticles and its effect on mechanical strength. Construction and Building Materials. 36:736-742. doi:10.1016/j.conbuildmat.2012.06.043.

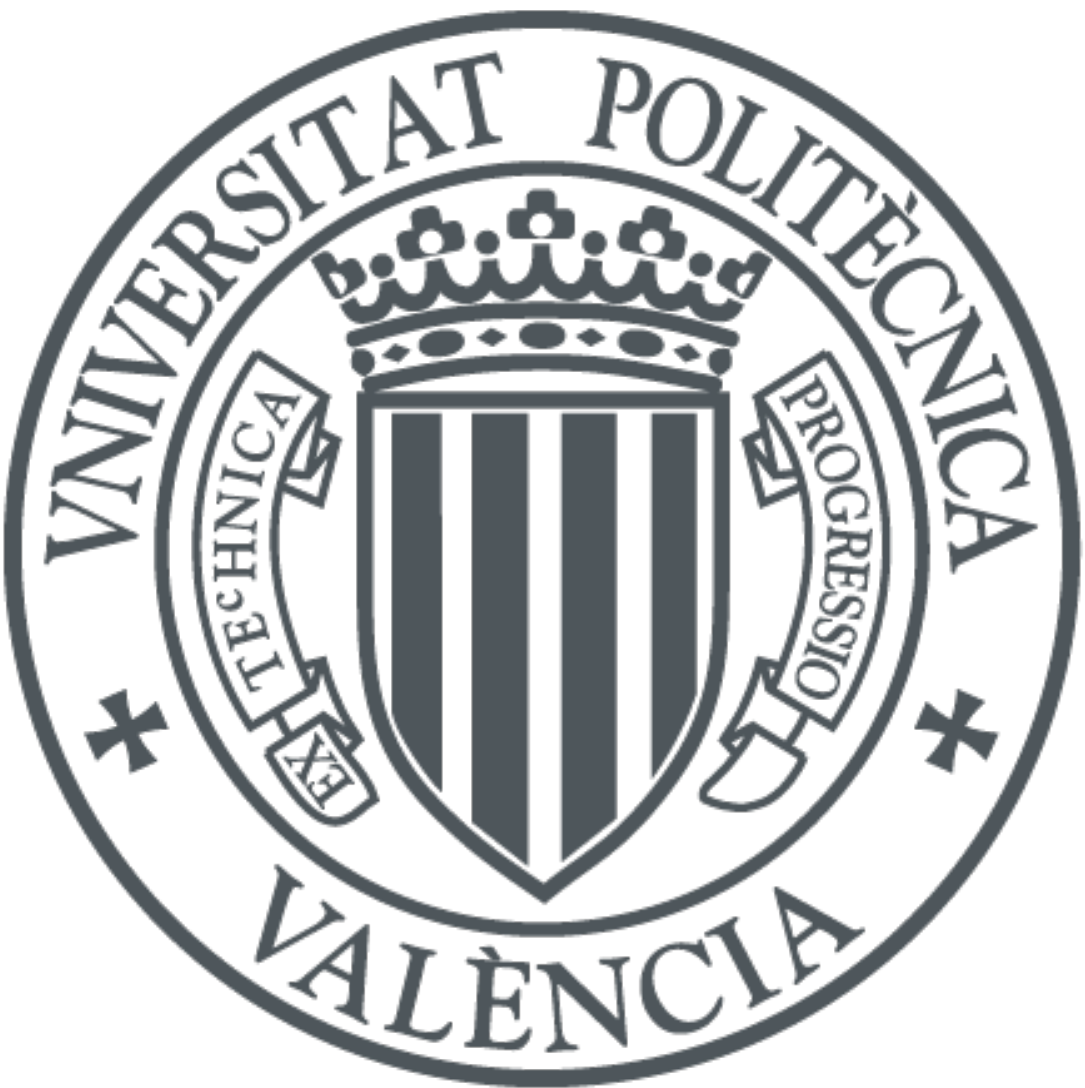

The final publication is available at

http://dx.doi.org/10.1016/j.conbuildmat.2012.06.043

Copyright Elsevier

Additional Information 


\title{
MINERALOGICAL EVOLUTION OF PORTLAND CEMENT BLENDED WITH SILICA NANOPARTICLES AND ITS EFFECT ON MECHANICAL STRENGTH
}

\author{
TOBÓN, J.I. ${ }^{1 *} ;$ PAYÁ, $J^{2} ;$ BORRACHERO, M. V. ${ }^{2} \&$ RESTREPO, O. J. \\ 1. Cement and Building Materials Group. Calle 75 \# 79a -51, Bloque M17-101, Escuela de Ingeniería de Materiales, \\ Facultad de Minas, Universidad Nacional de Colombia, Medellín \\ 2. Instituto de Ciencia y Tecnología del Hormigón, ICITECH. Universitat Politècnica de València. España
}

\begin{abstract}
Mineralogical analysis done on Spanish Portland cement Type I pastes, blended with nanosilica was carried out by conventional and high-resolution thermogravimetric analysis (TG-HRTG) and X-ray diffraction (XRD) in order to determine the quantity of the different mineralogical phases obtained during the hydration process. Simultaneously, mortars with the same materials and replacement ratio were made in order to assess their compressive strength for up to 28 days of curing time. In this paper, the rate and quantity of each one of the main constituent phases of the cement during its hydration process (C-S-H, ettringite, portlandite, stratlingite, etc.) were determined. A correlation between the quantity of C-S-H and the development of compressive strength was established. Additionally, the pozzolanic activity of nanosilica was evaluated by quantifying the fixation of calcium hydroxide and its impact on the development of the compressive strength.
\end{abstract}

KEYWORDS: HRTG, nanosilica, pozzolans, compressive strength, mineralogy of cements, blended cement

\section{INTRODUCTION}

Thermal analysis techniques are one of the most popularly used techniques for research on the physical and chemical phenomena associated with the formation and behavior of the hydrated phases of cement [1]. Traditionally, the quantification of hydrated phases with TG is done mainly on portlandite $[2,3]$ because of the fact that other hydrated phases show overlapping peaks since their decomposition temperatures are very close. This fact impedes a reliable identification of other hydrate phases like calcium silicate hydrates $\mathrm{C}-\mathrm{S}-\mathrm{H}$, ettringite, calcium aluminosilicate hydrates (CA-S-H), calcium aluminate hydrates $(\mathrm{C}-\mathrm{A}-\mathrm{H})$, etc. Using the modules of high-resolution in thermogravimetric analysis software (HRTG) the problem could be solved. These modules increase the resolution to reasonable testing times $[1,4-8]$ and can be applied successfully in the analysis of construction materials if the correct parameters are selected as was suggested by Tobón et al. [8]

With the use of HRTG, a new difficulty is found, due to the fact that the hydrated mineralogical phases of cement are not stoichiometric materials, it is common that the same phase show two or three peaks corresponding to different decomposition phenomena [8]. Because of this, a detailed study about the identification of cement-hydrated phases is necessary.

\footnotetext{
*Corresponding author: jitobon@unal.edu.co. Tel. \#(5-74) 425-5144, fax: (5-74) 425-5232
} 
Different factors are incidental in the development of the mechanical strength of hardened cement, such as: clinker composition and microstructure, cement particle size distribution, gypsum content and degree of gypsum hydration, the water/cement ratio, the curing time, the curing temperature and relative humidity, mineral additions and additives in the mixture, and mixing conditions, among others. But as Taylor pointed out [9] in different ways these factors also determine the phase's distribution and quantity in the cement matrix. Furthermore, these phases verify the microstructure of the hardened pastes. Hence, it is very important to find methods that allow for an accurate determination of the different hydrated phases in the cement pastes.

Nanosilica has proven to be an effective mineral addition for blending with Portland cement in order to improve the cement's mechanical performance $[10,11]$, and durability $[12,13]$ thanks to the its high pozzolanic activity, which enables to nanosilica to have a high lime-fixation capacity [11]. Researchers have found that nanosilica also accelerates the hydration reactions of $\mathrm{C}_{3} \mathrm{~S}$ in function of its characteristics [14,15]. But, the mineralogical evolution of cement pastes blended with nanosilica has not been quantified and the relationship to mechanical strength of mortars blended with it has not been found.

\section{MATERIALS AND METHODS}

Pastes were made with Spanish Portland cement Type I (52.5 MPa) blended with 0\%, 5\%, and 10\% of commercial nanosilica (Nyasil ${ }^{\mathrm{TM}} 5$ - Ny) produced by NYACOL, Nano Technology Inc. with $92 \%$ purity and a $\mathrm{d} 50=1.8 \mu \mathrm{m}$, water/cement-material $(\mathrm{w} / \mathrm{cm})$ of 0.4 . Ny has a low crystallinity grade and $98 \%$ purity. Pastes (control: Ce; $5 \%$ replacement $5 \mathrm{Ny} ; 10 \%$ replacement $10 \mathrm{Ny}$ ) were cured for $1,3,7,14$, and 28 days at $20^{\circ} \mathrm{C}$ in $100 \%$ relative humidity.

Mineralogical analysis was carried out with HRTG on a Mettler-Toledo TG 850 in aluminum crucibles of $100 \mu \mathrm{L}$ with a sealable lid perforated, in the presence of a self-generated atmosphere, and without purge gas in accordance with the optimal parameters found by Tobón et al. [8], which are shown in Table 1.

Table 1. Parameters chosen for the test by HRTG

\begin{tabular}{|l|c|}
\hline \multicolumn{1}{|c|}{ Parameter } & Value \\
\hline Amount of sample & $60 \mathrm{mg}$ \\
\hline Minimal heating rate & $0.5^{\circ} \mathrm{Cmin}^{-1}$ \\
\hline Maximum heating rate & $10^{\circ} \mathrm{Cmin}^{-1}$ \\
\hline Upper threshold & $3 \mu \mathrm{gs}^{-1}$ \\
\hline Lower threshold & $1 \mu \mathrm{gs}^{-1}$ \\
\hline Factor & 2 \\
\hline Waiting time & $6 \mathrm{~s}$ \\
\hline Temperature range & $35-300^{\circ} \mathrm{C}$ \\
\hline Purge gas & $0 \mathrm{mLmin}^{-1}$ \\
\hline
\end{tabular}

The conventional TG was done in a STA409 of the Netzsch, with a range between $20^{\circ}$ and $700^{\circ} \mathrm{C}$ and a heating rate of $10^{\circ} \mathrm{Cmin}^{-1}$. X-ray diffraction (XRD) was made in a PANalytical X'Pert PRO MPD, in a $2 \theta$ range between $2^{\circ}$ and $70^{\circ}$ with a step of $0.02^{\circ}$ and an accumulation time of $30 \mathrm{~s}$. 
In order to make some comparisons, we tested pastes of lime with nanosilica (Ny), nanoalumina (NA), and metakaolin (MK). The lime used was reagent calcium hydroxide with $96 \%$ purity from Riedel de Haën. We used nanoalumina Nyacol AL20SD with 94\% purity and a diameter of $50 \mathrm{~nm}$. The metakaolin used was Metastar 501 from IMERYS.

Mortars were conducted in accordance with UNE-EN 196-1 [16] in molds of 4x4x16 cm, with the same cement and the same nanosilica, in percentages equal to the percentages use for the replacement of pastes. Standardized sand was used. The $\mathrm{w} / \mathrm{cm}$ ratio was 0.5 , and the $\mathrm{arid} / \mathrm{cm}$ ratio was 3. Mortars remained in a curing room (at a saturation of $98 \%$ and $20^{\circ} \mathrm{C} \pm 2^{\circ} \mathrm{C}$ ) for 3 , 7, and 28 days.

\section{RESULTS AND DISCUSSION}

\subsection{MINERALOGY}

Using thermogravimetric analysis, the percentage of total mass loss (water from the hydrates) of the pastes was assessed at temperatures of up to $300^{\circ} \mathrm{C}$ and at different curing ages (Fig. 1). It was found that there is a greater total mass loss when the samples have a higher percentage of cement replacement by $\mathrm{Ny}$. This suggests an increase in the formation of hydrated mineral phases which are dehydrated at temperatures of up to $300^{\circ} \mathrm{C}(\mathrm{C}-\mathrm{S}-\mathrm{H}$, ettringite, $\mathrm{C}-\mathrm{A}-\mathrm{S}-\mathrm{H}$ and $\mathrm{C}-\mathrm{A}-\mathrm{H})$ by virtue of the quantity of nanosilica blended.

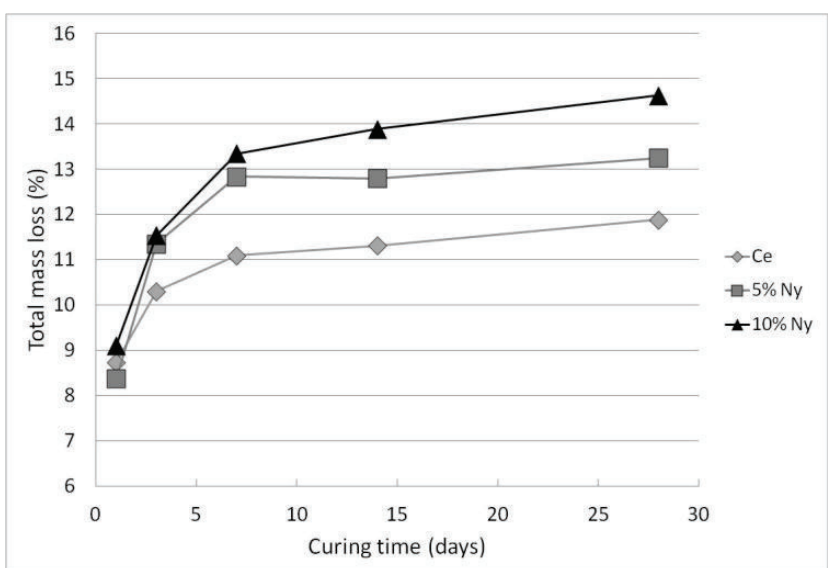

Figure 1. Mass loss from HRTG thermograms at temperatures of up to $300^{\circ} \mathrm{C}$

Figure 1 shows that on the first day there is no significant difference among the three pastes. To the seventh day of curing, the pastes with nanosilica ( $\mathrm{Ny}$ ) have a significantly greater mass loss. For the three samples remain the same trend and order in the mass loss after the seventh day. It can be interpreted as meaning that the impact of this mineral addition is minimal in the first hours of hydration. Ny becomes very important between the first and the seventh day of curing, producing a strong increase in the rate of hydration and in the quantity of hydrated phases formed.

Using HRTG, we conclude that when the percentage of replacement and the curing time are increased, the number of peaks in the thermograms is also increased because a larger and better formation of different mineral phases takes place (Figs. 2 and 3). Figure 2 shows the increase in the number of peaks in the derivative curves (DTG) from HRTG, with the curing time for $10 \%$ Nyasil- 
replaced paste. Figure 3 also shows a very good correlation among the peaks in the different pastes for the same curing age (28 days). All this allows us to propose a temperature range for each massloss event and thereby to achieve a better identification of the decomposition reactions for each phase. Seven temperature ranges were selected for this purpose.

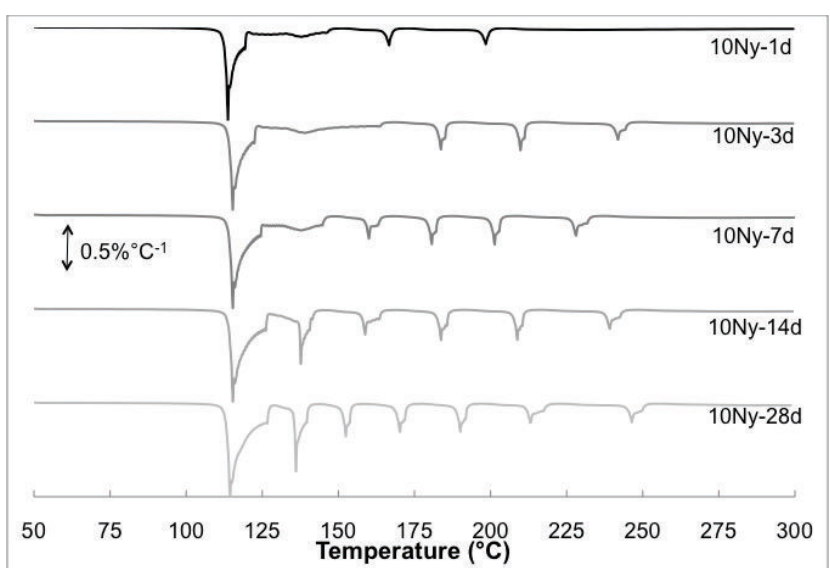

Figure 2. HRTG/DTG of pastes with $10 \%$ Ny until 28 curing days

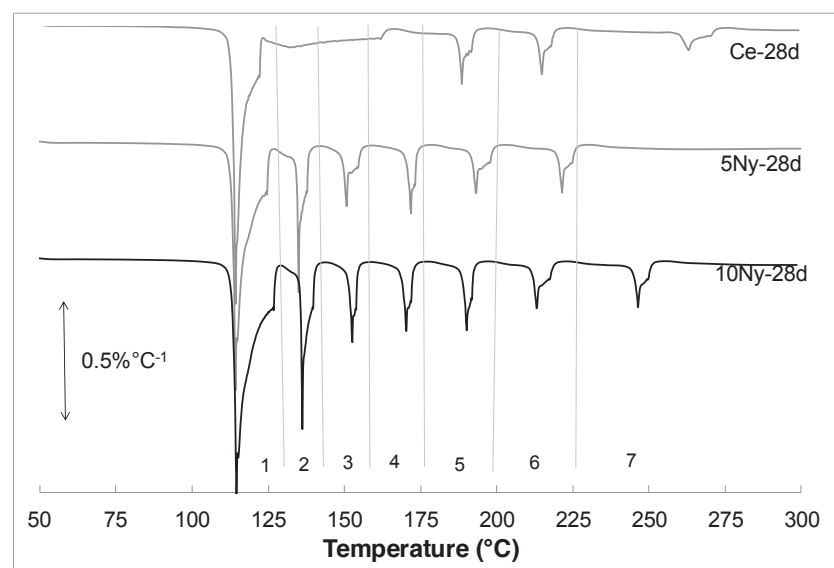

Figure 3. Temperature ranges from DTG curves among different pastes at the same curing age

3.1.1 Identification of peaks. To identify peaks present in the thermograms obtained with samples of cement with different percentages of replacement, reagent-grade hydrated lime $(\mathrm{CH})$ with $50 \%$ of mineral addition (Nyasil-Ny, metakaolin-MK, and nanoalumina-NA) were tested under the same conditions, in order to locate the decomposition temperatures of calcium silicate hydrate $(\mathrm{C}-\mathrm{S}-\mathrm{H})$, calcium aluminosilicate hydrate (C-A-S-H stratlingite), and calcium aluminate hydrate (C-A-H). The results obtained are presented in the Figure 4, the three DTG curves at the top of the figure correspond to cement samples cured for 14 days and the three DTG curves at the bottom are the samples of hydrated lime $(\mathrm{CH})$ with different replacement materials. 


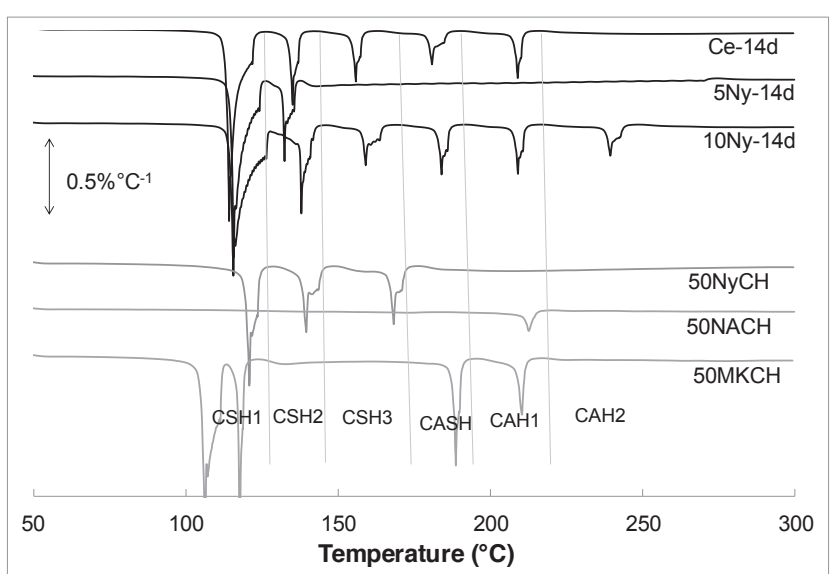

Figure 4. Identification of peaks obtained with HRTG

It was found that the first three peaks, from low to high temperature, correspond to the decomposition of calcium silicate hydrates (C-S-H), the fourth peak corresponds to calcium aluminosilicate hydrates (C-A-S-H), and the last two correspond to calcium aluminate hydrates (CA-H). This is because the first three peaks of the cement samples agree very well with those formed in the sample of hydrated lime with $\mathrm{Ny}$, where only calcium silicate hydrates can be formed (portlandite and calcite are another peaks that could be in this mixture, but they do not appear in the temperature range tested). The fifth peak in the cement samples agrees very well with the peak in the sample of lime with NA where only calcium aluminate hydrates can be formed. In the case of a sample of $\mathrm{CH}$ with MK calcium silicate hydrates, calcium aluminosilicate hydrates and calcium aluminate hydrates are expected to form, there are two peaks in the sample with MK that match well with those proposed for other samples such as C-S-H and C-A-H. Thus, the additional peak would be for the C-A-S-H. The last peak in the DTG of cement pastes with $10 \%$ of Ny partners with C-A-H because of the dehydration temperature that has been suggested for it by various authors [17].

Table 2 shows correlations between phases and the ranges of temperatures for the maximum decomposition rate $\left(\mathrm{T}_{\max }\right)$. The C-S-H2 peak coincides with the peak identified by several authors for the dehydration of ettringite [1], so it is possible to have an overlap of these two phases.

Table 2. Ranges of $\mathrm{T}_{\max }$ for each of the peaks identified

\begin{tabular}{|l|c|c|c|}
\hline Peak & $\mathbf{1}$ & $\mathbf{2}$ & $\mathbf{3}$ \\
\hline & $\left({ }^{\circ} \mathbf{C}\right)$ & $\left({ }^{\circ} \mathbf{C}\right)$ & $\left({ }^{\circ} \mathbf{C}\right)$ \\
\hline C-S-H & $90-125$ & $125-40$ & $140-165$ \\
\hline C-A-S-H & $165-185$ & & \\
\hline C-A-H & $185-215$ & $215-255$ & \\
\hline
\end{tabular}

\subsubsection{Behavior of the different mineral phases with curing time}

Calcium silicate hydrate (C-S-H). The appearance of the three peaks for the C-S-H phases in HRTG/DTG could be either due to different types of C-S-H, to transformations of C-S-H, or to the release of $\mathrm{OH}$ radicals from $\mathrm{C}-\mathrm{S}-\mathrm{H}$. To identify if each of these three C-S-H peaks correspond to 
different polymorphs of this mineral phase, or simply to different processes of decomposition within a single phase, XRD on the samples of paste of lime reagent were carried out (at $50 \%$ of replacement with $\mathrm{Ny}$ and $\mathrm{w} / \mathrm{cm}$ of 1.0 , at 3, 7, 14, and 28 days of curing time). Inside of the diffractometer, the temperature was varied: i.e. the same sample was studied at room temperature $\left(25^{\circ} \mathrm{C}\right)$, it was heated to 90,125 , and $150^{\circ} \mathrm{C}$, while XRD analysis were carried out for each of these temperatures. Just as the sample reached the selected temperature an XRD evaluation was made. The sample was left at that temperature for $10 \mathrm{~min}$ and then XRD was made again. All this was done in order to determine the changes produced on C-S-H by increasing of the temperature (Fig. $5)$.

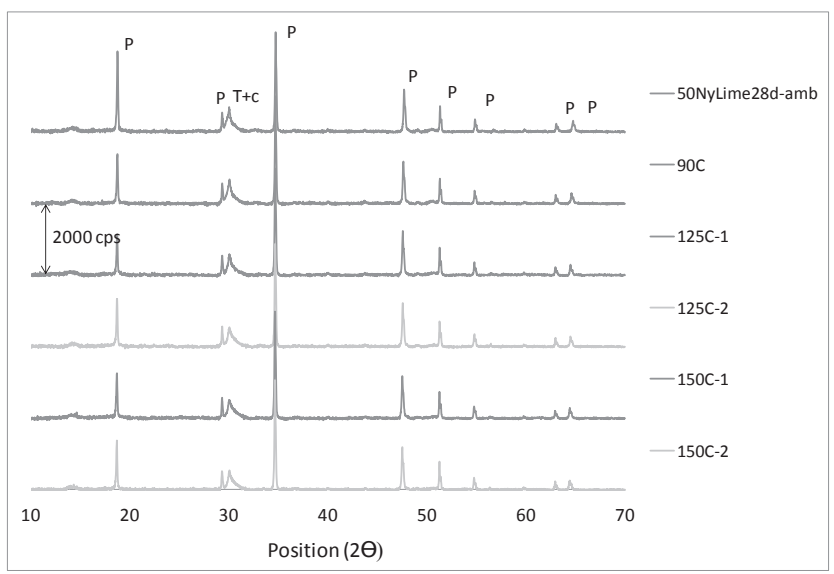

Figure 5. X-ray diffractograms of the 50NyLime samples at different temperatures; Legend: $\mathrm{P}$ (portlandite), T (C-S-H), and c (calcite)

In Figure 5, there is no evidence of the appearance or the disappearance of C-S-H peaks. By expanding the area between $28.5^{\circ}$ and $30^{\circ} 2 \theta$, where the main peak of the different types of C-S-H is located, it was found that for the same temperature there are no changes in the position of the peak (Fig. 6) and among the different temperatures, for the same sample, the peaks showed a decrease in their intensity, but no change in their position. This means that the three peaks found for C-S-H by HRTG do not correspond to polymorphs of this phase, but yes to different processes of decomposition. This interpretation agrees with the models proposed for $\mathrm{C}-\mathrm{S}-\mathrm{H}$, where water can be found between the mineral layers or in the form of $\mathrm{OH}^{-}$radicals, linked to tetrahedral silicon or to octahedral calcium [18-22]. 


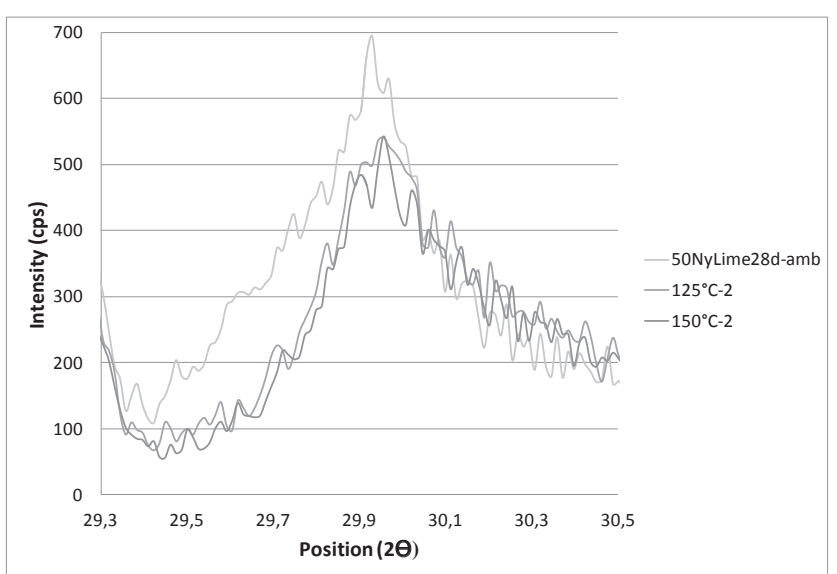

Figure 6. X-ray diffractograms of the $50 \mathrm{NyLime}$ samples treated at different temperatures

Due to what was explained in the last paragraph, it was decided that we would add the mass loss of the three peaks (Fig. 7) in order to assess the total water related to C-S-H. It was found that only for the first curing day there is a greater quantity of C-S-H for the control sample than for the blended samples. After three days and with growing trends, the samples with nanosilica showed a larger amount of C-S-H in comparison with the control sample, increasing the difference with the curing time and percentage of replacement. The amount of water associated with C-S-H is clearly related to the quantity of Ny in the pastes.

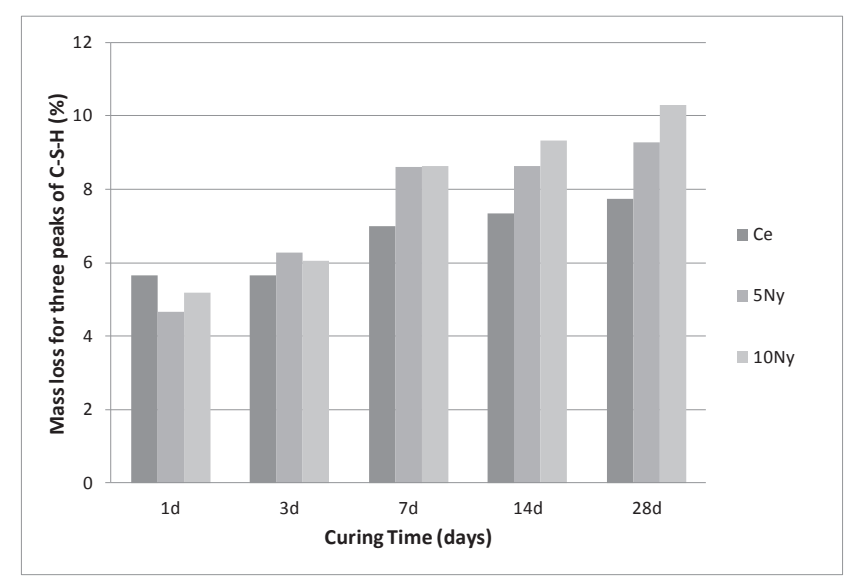

Figure 7. Percentage of mass loss for three C-S-H peaks

Along with the curing time the mass loss of C-S-H has a logarithmic behavior in samples of cement, with correlation coefficient between 0.85 and 0.95 (Fig. 8). 


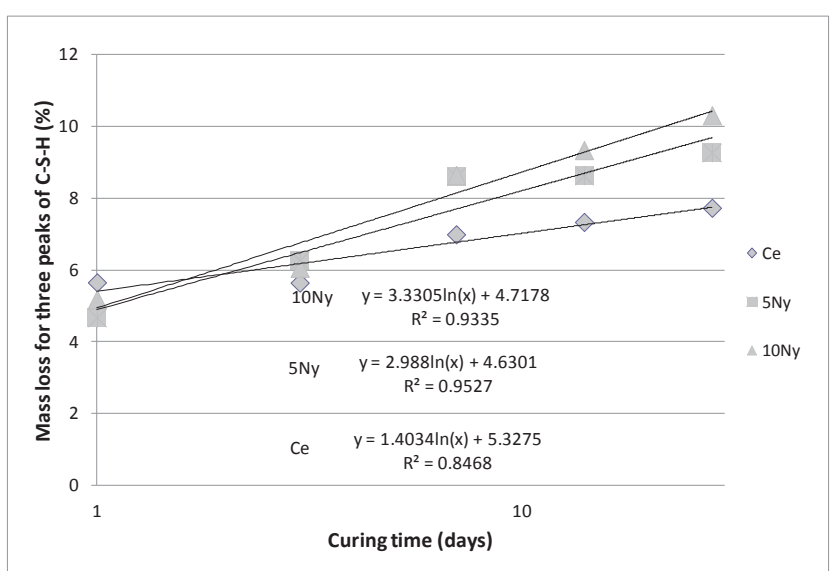

Figure 8. Modeling mass loss for C-S-H peaks with the time

Calcium aluminosilicate hydrates (C-A-S-H). This mineral phase was detectable only after the third day of hydration, and has a downward trend with the curing time, especially in blended cement (Fig. 9). Probably it was the presence of an additional $\mathrm{SiO}_{2}$ content in the mixture, which favored the transformation of C-A-S-H into a mixture of C-S-H and C-A-H.

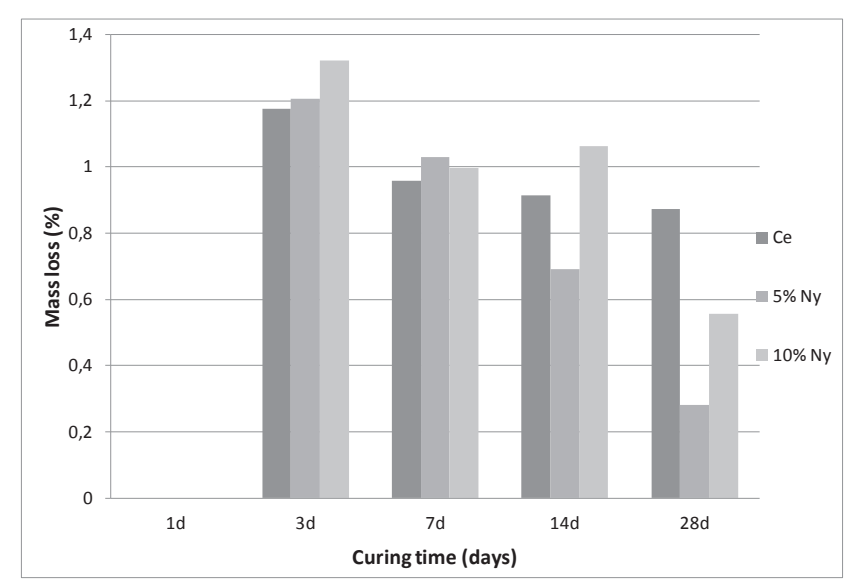

Figure 9. Mass loss of C-A-S- H peaks

Calcium aluminate hydrates $(\mathbf{C}-\mathbf{A}-\mathbf{H})$. Figure 10 shows the percentage of mass loss calculated on the C-A-H decomposition peaks, with curing time (as a sum of the two values). The C-A-H is always higher in the blended samples than in the control sample. This difference is greater for the first day of curing, and decreases along the curing time. This shows that the Ny has an accelerating effect on the cement hydration process. The trend is to have a higher content of $\mathrm{C}-\mathrm{A}-\mathrm{H}$ in the samples with $10 \% \mathrm{Ny}$, however, this content is very close to of the samples with $5 \% \mathrm{Ny}$. 


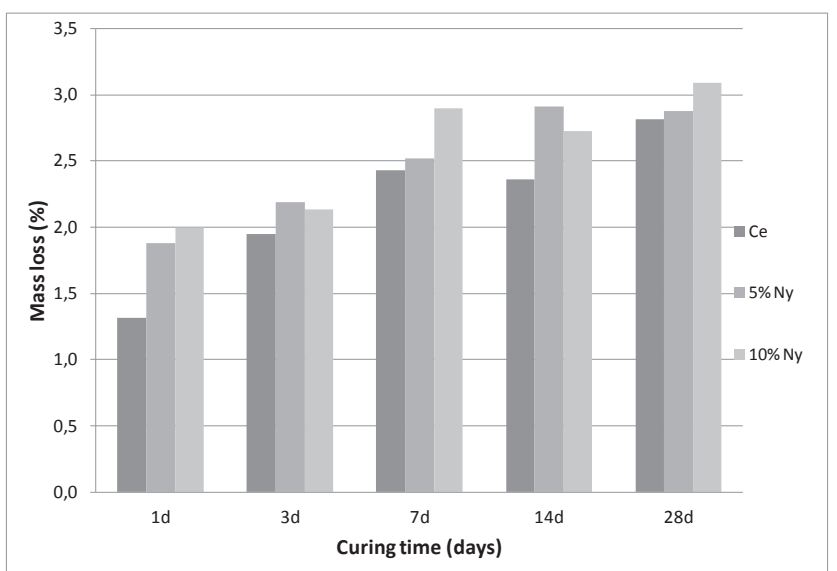

Figure 10. Mass loss of C-A-H peaks

\subsection{RELATIONSHIP BETWEEN MECHANICAL STRENGTH AND MINERALOGY}

The results of the compressive strength in mortars with $\mathrm{Ny}$ (Fig. 11) were correlated with the results of mineralogy in pastes (Fig. 12).

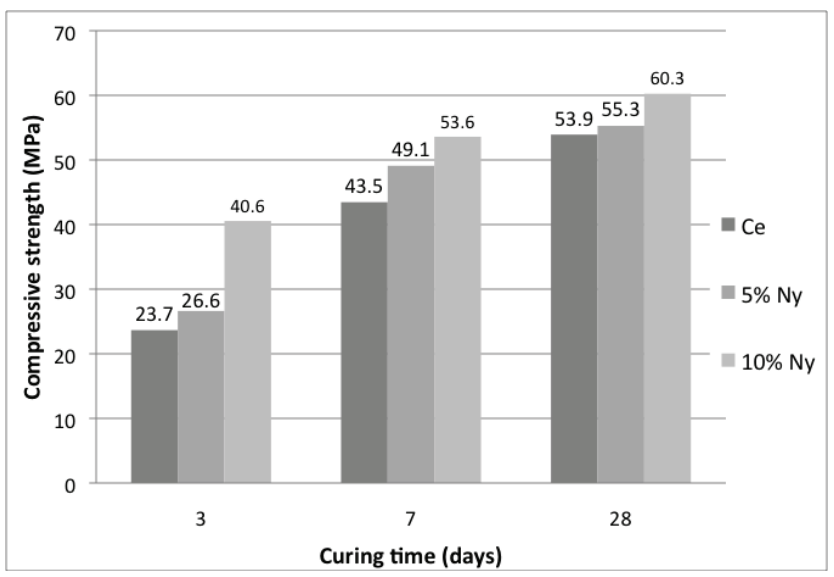

Figure 11. Compressive strength of mortars at 3, 7, and 28 curing days

Figure 11 shows that Ny has a positive effect on the development of the compressive strength (CS) of mortars, and that the highest CS values were obtained for $10 \% \mathrm{Ny}$ mortars. At three days of curing with $10 \%$ substitution, a $90 \%$ improvement was obtained. The effect of the mineral admixture is enormously positive, for example, to 7 curing days the sample with $10 \%$ Ny reached virtually the strength of the control sample to 28 curing days. These results show Ny to be an early activity pozzolan. 


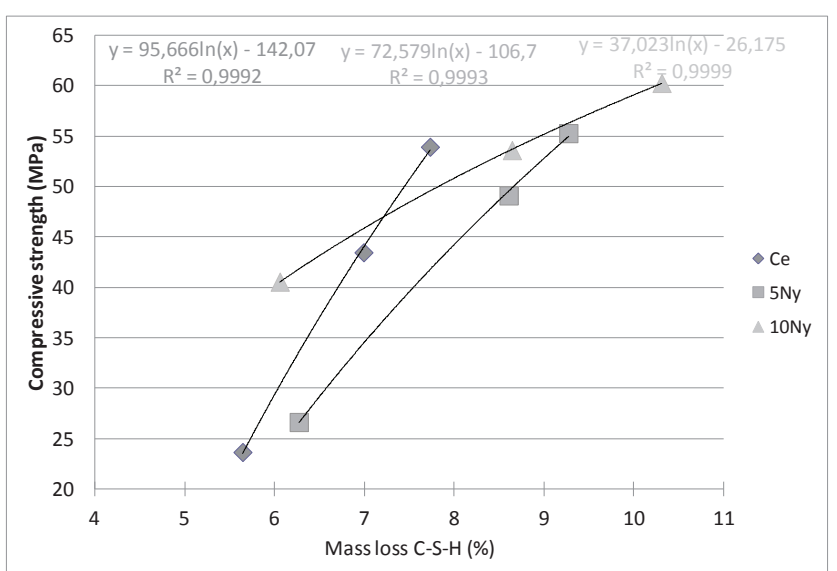

Figure 12. The relationship between the content of $\mathrm{C}-\mathrm{S}-\mathrm{H}$ in the pastes and the compressive strength for the mortars

Figure 12 shows that there is a very good correlation for each mixture $\left(R^{2}>0.99\right)$ between the content of C-S-H in the pastes (calculated by HRTG), and the mechanical strength developed by the mortars. In general, the increasing of $\mathrm{C}-\mathrm{S}-\mathrm{H}$ in the cementitious matrix is related to the higher compressive strength of the mortars.

It is interesting how at the beginning of the curves (point on left side of the Fig. 12), the samples with $10 \%$ Ny have a similar percentage of $\mathrm{C}-\mathrm{S}-\mathrm{H}$ than samples with $5 \% \mathrm{Ny}$, and have a much higher mechanical strength, which indicates that at initial ages it is not only the amount of C-S-H which explains compressive strength. Surely, in addition to the amount of C-S-H, the effective distribution of C-S-H plays an important role on mechanical properties.

At the early curing age, C-S-H quantity is not the only factor which explains compressive strength, but to final ages of hydration the C-S-H quantity is clearly the main parameter on the control of compressive strength. This is why when all the dates are assessed together, the correlation coefficient $\left(\mathrm{R}^{2}\right)$ drops, but it continues to be acceptable $\left(\mathrm{R}^{2}=0.82\right)$. It can be seen that the dispersion of the date is larger at earlier ages.

Likewise, in the Fig. 13 one can see that to early curing ages a higher lime fixation is equivalent to higher compressive strength, but to final ages the relationship is not in this way. 


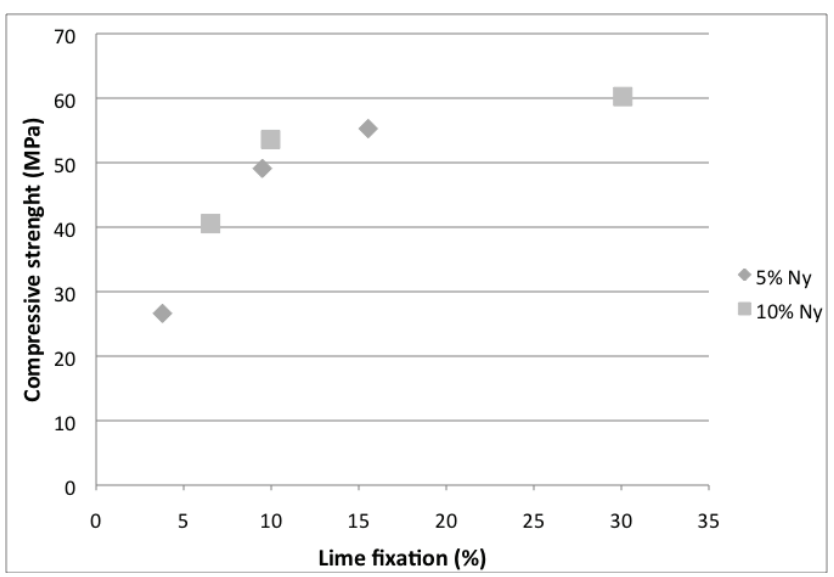

Figure 13. Relationship between the lime fixation in the pastes and the compressive strength of the mortars

The previous statement means that at the initial age, compressive strength of mortars depends mainly on the mineralogy and to a lesser degree on other factors such as the quantity of nucleation centers and how well-distributed they might be in the cementitious matrix. At the final age compressive strength depends almost exclusively on the percentage of C-S-H present.

\section{CONCLUSIONS}

- HRTG showed itself to be a powerful tool for the identification and quantification of the cement hydrated phases (C-S-H, C-A-S-H, C-A-H). This technique allows for one to improve the resolution so that it may avoids overlapping of peaks, which are commonly drawn in the thermograms of these phases. It was possible to determine the range of temperature where these phases were decomposed.

- There is a larger quantity of hydrates in the pastes as a result of increasing the percentage of substitution of cement by Ny, especially, from the first day of curing.

- C-S-H content has a logarithmic behavior along of curing time with a correlation coefficient between 0.85 and 0.95 ; except for when it is at first day of curing. Blended samples always have a longer quantity of this phase with regard to the plain sample.

- At 28 curing days with the $5 \%$ and the $10 \%$ of cement substitution by $\mathrm{Ny}$, the increase in C-S$\mathrm{H}$ content is close to $20 \%$ and $34 \%$, respectively.

- There is a very good correlation $\left(\mathrm{R}^{2}>0.99\right)$ between C-S-H content and mechanical strength developed by the mortars with different percentages of substitution in the curing time.

- To early ages, there is a small improvement in the quantity of C-S-H, but it has a good distribution in the matrix, which represents a noticeable improvement in compressive strength.

\section{ACKNOWLEDGMENTS}

The authors express their thanks to Cementos Argos S.A. and to COLCIENCIAS (Project 20201007768) for their financial support in the execution of this research.

\section{REFERENCES}

1. V. S. Ramachandran, R. M. Paroli, J. J. Beaudoin \& A. H. Delgado. Thermal analysis of construcción materials. Building Materials Series. Noyes Publications, New York, 2003. 679p. 
2. J. Payá, J. Monzó, M.V. Borrachero, S. Velázquez, M. Bonilla. Determination of the pozzolanic activity of fluid catalytic cracking residue. Thermogravimetric analysis studies on FC3R-lime pastes. Cem Concr Res 2003; 33:1085-1091

3. Ivindra Pane, Will Hansen. Investigation of blended cement hydration by isothermal calorimetry and thermal analysis. Cem Concr Res 2005; 35:1155- 1164

4. R. Riesen. Adjustement of heating rate for maximun resolution in TG and TMA (MaxRes). J Therm Anal Calorim 1998; 53:365 - 374.

5. P. Gill, S. Sauerbrunn and B. S. Crowe. High resolution thermogravimetry 1992; 38:255-266.

6. M. V. Borrachero, J. Payá, M. Bonilla and J. Monzó. The use of thermogravimetric analysis technique for the characterization of construction materials: The gypsum case. J Therm Anal Calorim 2008; 91(2):503-509.

7. J-F. Masson, Sladana Bundalo-Perc. Solventless fingerprinting of bituminous materials: A high-resolution Thermogravimetric method. Thermochimica Acta 436 (2005) 35-42.

8. J. I. Tobón, J. Payá, M. V. Borrachero, L. Soriano \& O. J. Restrepo. Determination of the optimum parameters in the high resolution thermogravimetric analysis (HRTG) for cementitious materials. J Therm Anal Calorim. (2012) 107:233-239.

9. H. F. W. Taylor. Cement Chemistry. 2nd edition. Thomas Telford, London, 1997. 459p.

10. J. I. Tobón, O. J. Restrepo \& J. Payá. Comparative analysis of performance of Portland cement blended with nanosiliaca and silica fume. Dyna, year 77, No 163, pp 37-46. Medellín, september 2010. ISSN 0012-7353.

11. Ye Qing, Zhang Zenan, Kong Deyu, Chen Rongshen. Influence of nano-SiO2 addition on properties of hardened cement paste as compared with silica fume. Construct Build Mater 2007; 21:539-545.

12. Ji T. Preliminary study on the water permeability and microstructure of concrete incorporating nano-SiO2. Cem Concr Res 2005;35(10):1943-7.

13. Gaitero JJ, Campillo I, Guerrero A. Reduction of the calcium leaching rate of cement paste by addition of silica nanoparticles. Cem Concr Res 2008;38(8-9):1112-8.

14. Jo B-W, Kim C-H, Tae G-h, Park J-B. Characteristics of cement mortar with nano-SiO2 particles. Construct Build Mater 2007;21(6):1351-5.

15. Florence Sanchez \& Konstantin Sobolev. Nanotechnology in concrete - A review. Construct Build Mater 2010; 24: 2060-2071.

16. UNE-EN 196-1. "Métodos de ensayo de cementos. Parte I: Determinación de resistencias mecánicas". 1996.

17. Ramachandran, V.S.; Paroli, R.M.; Beaudoin, J.J. \& Delgado, A.H. Thermal analysis of construction materials. Building Materials Series. Noyes Publications, New York, 2003.

18. Dolado, J. S.; Griebel, M. and J. Hamaekers. A Molecular Dynamics Study of Cementitious Calcium Silicate Hydrate (C-S-H) Gels. Journal of the American Ceramic Society: 90, 39383942, 2007.

19. Richardson, I.G. The calcium silicate hidrates. Cem Concr Res 38 (2008) 137-158.

20. Nonat, A. The structure and stoichiometry of C-S-H, Cem Concr Res 2004; 34:1521-1528.

21. Richardson, I. G. and Groves, G. W. Models for the composition and structure of calcium silicate hydrate $(\mathrm{C}-\mathrm{S}-\mathrm{H})$ gel in hardened tricalcium silicate pastes, Cem Concr Res 1992; 22(6):1001-1010.

22. Pellenq, R.; Kushima, A.; Shahsavari, R.; Van Vliet, K.; Buehler, M.; Yip, S. and Ulm, F. A realistic molecular model of cement hydrates. Proceedings of the National Academy of Sciences of the United States of America: 106, 16102-16107, 2009. 